Research Article

\title{
Numerical Modeling of Coupled Surge-Heave Sloshing in a Rectangular Tank with Baffles
}

\author{
Lv Ren, ${ }^{1,2}$ Yinjie Zou, ${ }^{3,4}$ Jinbo Tang, ${ }^{5}$ Xin Jin $\mathbb{C D}^{3,4}$ Dengsong Li, ${ }^{6}$ and Mingming Liu ${ }^{3}$ \\ ${ }^{1}$ College of Hydraulic \& Environmental Engineering, China Three Gorges University, Yichang, Hubei 443000, China \\ ${ }^{2}$ China Renewable Energy Engineering Institute, Beijing 100120, China \\ ${ }^{3}$ College of Energy, Chengdu University of Technology, Chengdu 610059, China \\ ${ }^{4}$ State Key Laboratory of Hydraulic Engineering Simulation and Safety, Tianjin University, Tianjin 300072, China \\ ${ }^{5}$ Key Laboratory of Mountain Hazards and Surface Process, Institute of Mountain Hazards and Environment, \\ Chinese Academy of Sciences, Chengdu 610041, China \\ ${ }^{6}$ College of Water Conservancy and Hydropower Engineering, Sichuan Agricultural University, Ya-an, Sichuan 625014, China
}

Correspondence should be addressed to Xin Jin; jinx@cdut.edu.cn

Received 6 January 2021; Revised 5 April 2021; Accepted 11 May 2021; Published 1 June 2021

Academic Editor: Mi-An Xue

Copyright (C) $2021 \mathrm{Lv}$ Ren et al. This is an open access article distributed under the Creative Commons Attribution License, which permits unrestricted use, distribution, and reproduction in any medium, provided the original work is properly cited.

\begin{abstract}
Liquid sloshing under coupled surge and heave excitations in a rectangular tank has been numerically investigated by applying a Navier-Stokes solver. Fieriest coupled sloshing was further considered, and the internal baffle was expected to suppress the violent sloshing wave. After getting fully validated against available results from the literatures, the numerical model was applied to research coupled sloshing, and both vertical baffle and horizontal baffle have been considered. Due to the strong vortexes created by the sharper corners of the baffles and the reduction of the effective water bulk climbing through the tank walls, the sloshing was dramatically reduced. The increase of the baffle distance away from the tank bottom led to a decrease in the sloshing wave. It was noted that the baffle near the free surface caused the maximal dissipation. The frequency response of the sloshing wave was accordingly illustrated.
\end{abstract}

\section{Introduction}

Liquid sloshing widely exists in partially filled tanks encountering external excitations, such as seismic excitation, ship motion, road transportation, and so on. Good prediction of this phenomenon is of great engineering value and critical for the tank design. The triggering condition of the violent sloshing, which can result in strongly localized loads, is the main concern in engineering applications. Most researches aimed at explaining the sloshing wave excited by one-degree-of-freedom excitation and correlated damping approaches; relatively a few papers focused on the coupled ones. As a result of the frequently reported incidents caused by the liquid sloshing [1], it is necessary to study coupled sloshing and find effective ways to mitigate the sloshing energy to ensure structural safety.
Some theoretical approaches such as separation of variables, perturbation, and multimodal methods have been applied to study the linear and nonlinear sloshing waves [2-6]. A third-order nonlinear solution for the surge-excited sloshing was derived by Faltinsen [7]. Benjamin and Ursell [8] obtained a linear solution for heave-excited sloshing. Frandsen [4] applied the perturbation method to identify the responses of the coupled surge-heave excited sloshing, and the excitation conditions of the coupled resonant sloshing were given. In view of the nonlinear sloshing wave, Faltinsen et al. [9-11] did a series of analytical studies. Further work on the strongly nonlinear coupled sloshing is still needed.

If the excitation frequency is close to the system's natural frequencies, and the excitation amplitude is reasonably large, the sloshing wave is prone to break; those analytical solutions can be completely invalid [12-15]. The numerical 
methods become popular as they are effective supplements to realize the nonlinear phenomenon. A coupled finite element method (FEM) and boundary element method (BEM) solver was developed to research the incompressible and nonlinear wave motions in confined tanks under external excitations [16,17]. Godderidge et al. [18] applied a commercial computational fluid dynamics (CFD) model (Fluent) to investigate nonlinear sloshing. The Navier-Stokes solver was used to predict wave and pressure responses in tanks with various shapes [19].

Except for the high-profile sloshing waves excited by one-degree-of-freedom excitation, the multi-degree-offreedom excitation is also critical in engineering applications, but relatively less attention has been paid to it. Nevertheless, several studies guided us in realizing this phenomenon. Following the early analytical studies of Faltinsen [2] and Benjamin and Ursell [8], Frandsen [4] gave a third-order solution for coupled surge-heave excited sloshing, which has been verified by Ning et al. [20] through a boundary element numerical model. More complex sloshing excited by two- or three-degree-of-freedom excitations (surge + pitch and surge + pitch + heave) was illustrated by Chen and Nokes [21]. The sloshing waves under other combing forms of external excitations, such as surge + heave + roll and surge + pitch have also been shown by Bai et al. [22] and Luo et al.[23], respectively. They concluded that the most violent case could be aroused when the excitation frequencies of all degrees of freedom were close to the system's natural frequencies.

In order to reduce the sloshing intensity and ensure the system's stability, the arrangement of internal baffles has yet been proved to be an effective way [5,24]. Specifically, the vertical and horizontal baffles were applied to suppress nonlinear or breaking sloshing to ensure safety [20,25-28]. Akyildiz and Ünal [29] explained the effectiveness of the vertical and horizontal baffles on reducing nonlinear sloshing under pitch excitations. Xue et al. [30] declared the vertical baffle can shift the system's natural frequencies away from that of the bare tank, leading to sloshing being offresonant. Recently, the OpenFOAM numerical model becomes popular [31-33]. Sanapala et al. [34] explained the damping effects of the horizontal baffles on heave-excited sloshing in a rectangular tank. Saghi et al. [35] gave an optimization arrangement of the vertical baffles by the criterion of the entropy generation. The vertical, horizontal, and combined vertical and horizontal baffles have been systematically simulated that have been verified to be useful tools to damp the sloshing wave under seismic excitations [36].

Even though the suppression of sloshing under onedegree-of-freedom excitations has been reported extensively, to the best of our knowledge, less attention has been drawn to the suppression of sloshing under multiple excitations. To address this knowledge gap, the coupled surgeheave excited sloshing was investigated through a NavierStokes solver; both vertical and vertical baffles were applied to reduce coupled sloshing. The baffle position and configuration were discussed in detail. The damping mechanism was demonstrated and discussed. The rest of this paper was organized as follows: (1) the numerical methodology and validation were shown in section 2; (2) discussions and results were given in section 3; (3) conclusions and main findings of the work were presented in section 4 .

\section{Numerical Methodology and Validation}

The incompressible Newtonian fluids (water and air) are considered, which can be described through the NavierStokes equations. The structure is modeled by the virtual boundary force (VBF) method [12], and the free surface is tracked by the volume-of-fluid (VOF) method [37]. This section is divided into three parts: one introduces the basic governing equations, and other two give the validations.

2.1. Basic Governing Equations. The basic governing equations for water and air are the conservation of mass and momentum equations, namely, the continuity equation and the spatially averaged Navier-Stokes equations (SANS), which are summarized as follows:

$$
\begin{aligned}
\frac{\partial u_{j}}{\partial x_{j}} & =0 \\
\frac{\partial u_{i}}{\partial t}+u_{j} \frac{\partial u_{i}}{\partial x_{j}} & =-\frac{1}{\rho} \frac{\partial p}{\partial x_{i}}+g_{i}+\frac{1}{\rho} \frac{\partial \tau_{i j}}{\partial x_{j}}+\frac{1}{\rho} \frac{\partial \tau_{i j}^{r}}{\partial x_{j}}+\left(f_{V B F}\right)_{i},
\end{aligned}
$$

where $i, j=1,2,3$ for three-dimensional flows; $u_{i}$ is the filtered velocity; $p$ stands the filtered pressure; $t$ is the time; $\rho$ is the density; and $g_{i}$ represents the body force. $\tau_{i j}$ and $\tau_{i j}^{r}$ denote the molecular viscous and SGS Reynolds stress tensors, respectively. $\tau_{i j}^{r}=2 \rho v_{t} \sigma_{i j}$, where $\sigma_{i j}$ is the strain rate; $v_{t}$ represents the molecular eddy viscosity $l_{s}^{2} \sqrt{\left(2 \sigma_{i j} \sigma_{i j}\right)}$, where $l_{s}$ is formulated as the product of the Smagorinsky length scale $C_{s}(0.15)$, recommended by [37], and the filter width $\sqrt[3]{\Delta x \Delta y \Delta z}$, among which $\Delta x, \Delta y$, and $\Delta z$ are the grid lengths in the three directions. $\left(f_{V B F}\right)_{i}$ denotes the reaction force between the fluid and solid. In this study, the translational motions (horizontal and vertical movements) are considered; hence, $g_{i}$ not only includes the gravitational acceleration but also contains the translational acceleration.

The water-air interface is tracked by the VOF method, which is defined as follows:

$$
\frac{\partial F}{\partial t}+u_{i} \frac{\partial F}{\partial x_{i}}=0
$$

where $F=\left(\rho-\rho_{a}\right) /\left(\rho_{w}-\rho_{a}\right)$ is the volumetric fraction between air and water, which is equal to 0 in air and 1 in water, and $\rho_{a}$ and $\rho_{w}$ are the densities of air and water, respectively.

The finite difference method is applied to discretize the governing equations on the staggered grid system. The twoprojection method is employed to solve the momentum equation as follows: 


$$
\begin{aligned}
& \frac{\widetilde{u}_{i}^{n+1}-u_{i}^{n}}{\Delta t}=-u_{j}^{n} \frac{\partial u_{i}^{n}}{\partial x_{j}}+\frac{\partial}{\partial x_{j}}\left[\frac{1}{\rho^{n}} \frac{\partial\left(\tau_{i j}\right)^{n}}{\partial x_{j}}+\frac{1}{\rho} \frac{\partial\left(\tau_{i j}^{r}\right)^{n}}{\partial x_{j}}\right], \\
& \frac{u_{i}^{n+1}-\widetilde{u}_{i}^{n+1}}{\Delta t}=-\frac{1}{\rho^{n}} \frac{\partial p^{n+1}}{\partial x_{i}}+g_{i}^{n+1}+\left(f_{V B F}\right)_{i}^{n+1},
\end{aligned}
$$

where $\widetilde{u}_{i}^{n+1}$ is an intermediate velocity at the $(n+1)$ th time step.

The $\left(f_{V B F}\right)_{i}$ is defined as follows:

$$
\left(f_{V B F}\right)_{i}^{n+1}= \begin{cases}\frac{u_{i}^{n+1}-\widetilde{u}_{i}^{n+1}}{\Delta t}+\frac{1}{\rho^{n}} \frac{\partial p^{n+1}}{\partial x_{i}}-g_{i}^{n+1}, & \text { fluid - solid interface } \\ 0, & \text { else where. }\end{cases}
$$

In order to guarantee the no-slip velocity at the fluidsolid interface, a new velocity $\widehat{u}_{i}^{n+1}$ is adopted to replace $u_{i}^{n+1}$, which is interpolated by the intermediate velocity in the interior fluid cell and the no-slip velocity on the fluid-solid interface. $\widehat{u}_{i, j, k+1 / 2}^{n+1}$ is taken, for example, as shown in Figure 1, which can be formulated as

$$
\widehat{u}_{i+1 / 2, j, k}^{n+1}=\frac{\delta z}{\left(\Delta z_{k}+\Delta z_{k+1}\right) / 2+\delta z} \widetilde{u}_{i, j, k+1 / 2}^{n+1} .
$$

2.2. Coupled Sloshing in a Bare Tank. Sloshing under coupled surge-heave excitations has been numerically investigated by Frandsen [4]. A two-dimensional tank with length $L=1.0 \mathrm{~m}$ and mean water depth $h=0.5 \mathrm{~m}$ was considered. According to the dispersion relationship $\omega_{n}^{2}=g k_{n} \tanh \left(k_{n} h\right)$, where $k_{n}=n \pi / L, g=9.81 \mathrm{~m} / \mathrm{s}^{2}$, and $n=1,2,3 \cdots$, the first-order natural frequency $\omega_{1}$ is equal to $5.3166 \mathrm{rad} / \mathrm{s}$. The horizontal and vertical displacements of the tank followed $x(t)=$ $a_{\mathrm{h}} \cos \left(\omega_{\mathrm{h}} t\right)$ and $z(t)=a_{\mathrm{v}} \cos \left(\omega_{\mathrm{v}} t\right)$, respectively, among which $a_{\mathrm{h}}=0.000506 \mathrm{~m}, \omega_{\mathrm{h}}=0.98 \omega_{1}, a_{\mathrm{v}}=0.2724 \mathrm{~m}$, and $\omega_{\mathrm{v}}=0.8 \omega_{1}$. The uniform grid system $200 \times 200$ is used in the present simulation, with the grid sizes $\Delta x=0.0025 \mathrm{~m}$ and $\Delta z=0.0005 \mathrm{~m}$.

The time histories of the free surface displacements at the left wall are shown in Figure 2(a). All the three results agree rather well at the beginning nondimensional time $t \times \omega_{1}=50$; considering that the surge excitation frequency is close to the first-order natural frequency, both numerical results gradually derivate from the analytical solution with the increasing wave steepness due to the resonance. The phase-plane charts of the analytical solution and present numerical result are presented in Figures 2(b) and 2(c), respectively. The analytical result displays a linear behavior-symmetrical trough and crest. As for the numerical one, there exist apparent differences between trough and crest, and the absolute value of the crest is larger than that of the trough, displaying an obviously nonlinear phenomenon.

2.3. Sloshing under Surge Excitation in a Baffled Baffle. Xue et al. [30] conducted a series of table experiments to reveal the damping effects of baffles in suppressing sloshing under surge excitations in a three-dimensional tank with length $L=0.57 \mathrm{~m}$ and width $W=0.31 \mathrm{~m}$. A vertical baffle with $0.1 \mathrm{~m}$ in height and $0.006 \mathrm{~m}$ in thickness was arranged in the middle of the tank in the length direction, as shown in Figure 3 . The mean water depth of $h=0.18 \mathrm{~m}$ was adopted. The tank displacement at length direction followed the cosine function: $x=-a \cos (\omega t)$, of which the displacement amplitude and external excitation frequency were $a=0.02 \mathrm{~m}$ and $\omega=6.403 \mathrm{rad} / \mathrm{s}$, respectively.

In the present simulation, the computational domain $0.57 \mathrm{~m} \times 0.31 \mathrm{~m} \times 0.25 \mathrm{~m}$ is discretized into $95 \times 62 \times 50$ uniform grids, with the grid sizes $\Delta x=0.006 \mathrm{~m}$ and $\Delta y=\Delta z=0.005 \mathrm{~m}$. The dynamic pressures at $0.065 \mathrm{~m}(P 1)$ and $0.185 \mathrm{~m}(P 2)$ away from the right tank wall are shown in Figure 4. It can be found that the present numerical results agree rather well with the experimental data of Xue et al. [30], especially in phase. There exist some discrepancies in the peak, which may be mainly caused by the slightly breaking free surface leading to some uncertainty arising from air entrainment.

\section{Results and Discussion}

This part discusses the coupled surge-heave excited sloshing in baffled tanks. Two kinds of baffles, namely, vertical and horizontal baffles, are arranged in the tanks with the expectation to reduce the sloshing wave. A three-dimensional rectangular tank with length $L$, width $W$, and mean water depth $h$ of $0.49 \mathrm{~m}, 0.1 \mathrm{~m}$, and $0.3 \mathrm{~m}$, respectively, is used. Therefore, the first-order natural frequency of the partially filled tank is $\omega_{1}=7.763 \mathrm{rad} / \mathrm{s}$. According to Frandsen and Peng [38] and Jin [39], the most adverse coupled surgeheave excited sloshing is the case that the surge excitation frequency is close to the first-order natural frequency, and the heave excitation is equal to twice of the first-order natural frequency. The horizontal and vertical displacements satisfy the cosine functions $x(t)=a_{\mathrm{h}} \cos \left(\omega_{\mathrm{h}} t\right)$ and $z(t)$ $=a_{\mathrm{v}} \cos \left(\omega_{\mathrm{v}} t\right)$, respectively, where $a_{\mathrm{h}}=0.008 \mathrm{~m}, \omega_{\mathrm{h}}=\omega_{1}$, $a_{\mathrm{v}}=0.005 \mathrm{~m}$, and $\omega_{\mathrm{v}}=2.0 \omega_{1}$.

3.1. Baffle Arrangements. The vertical and horizontal baffles considered in the present study are shown in Figures 5(a) and 5(b), respectively. As for the vertical baffle, $H v$ and $D v$ represent the height of the vertical baffle and distance from 

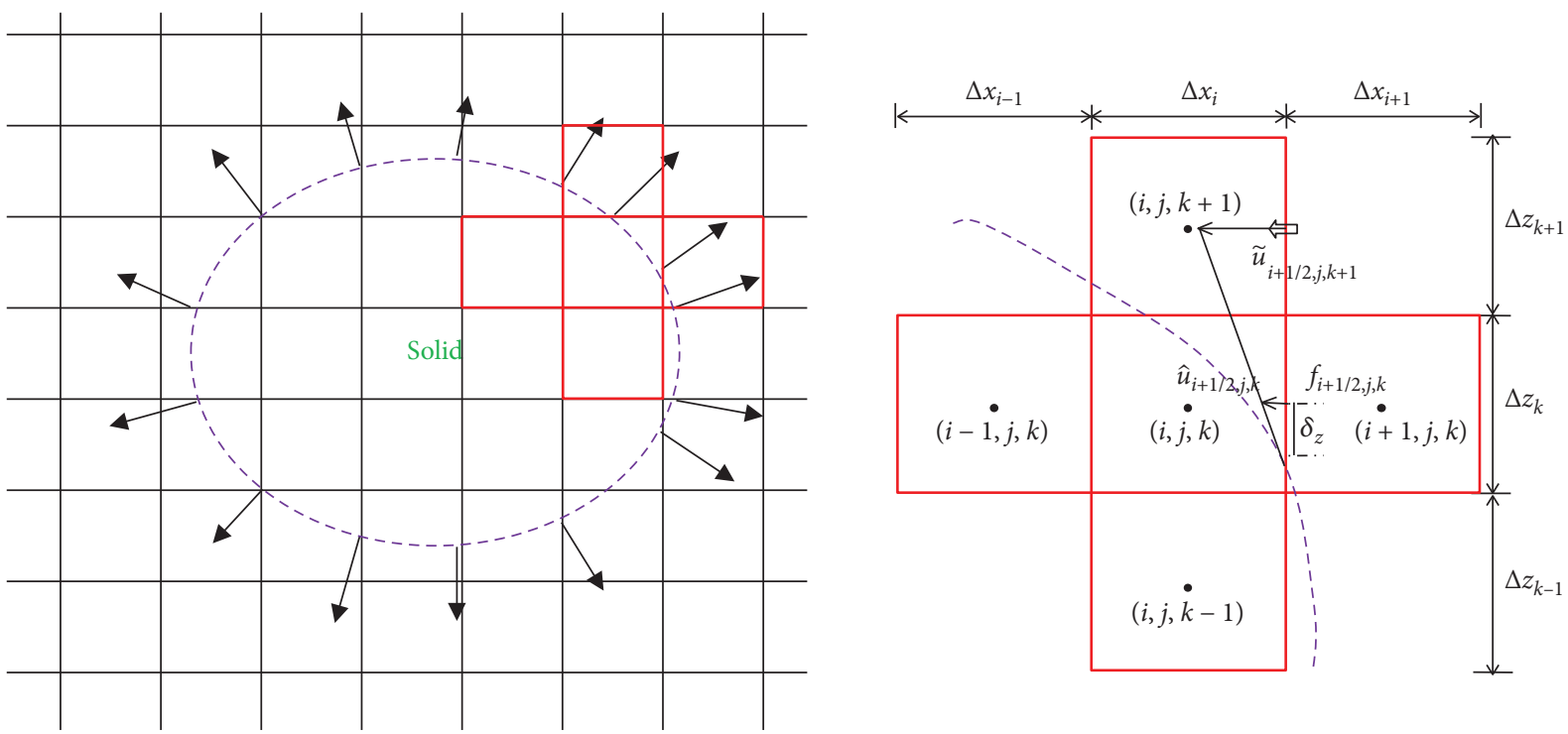

FIGURE 1: Schematical map of the VBF method.

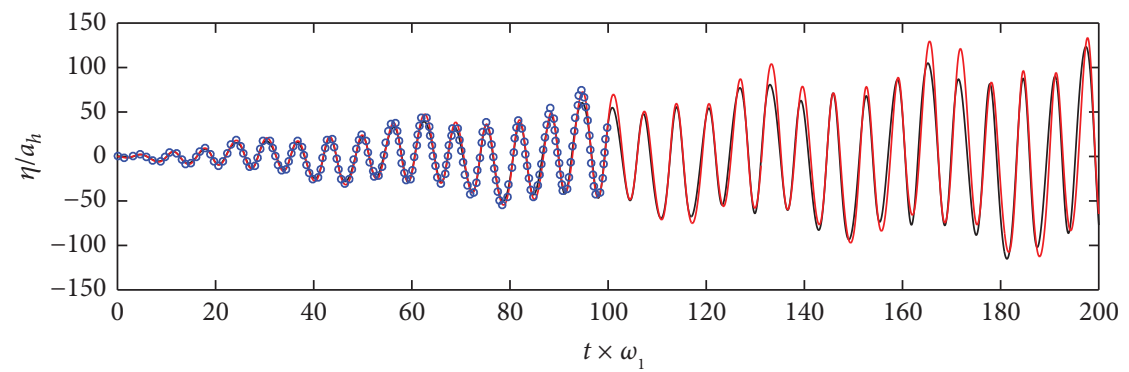

- Analytical solution

- - - Present numerical result

- Numerical result of Frandsen [4]

(a)

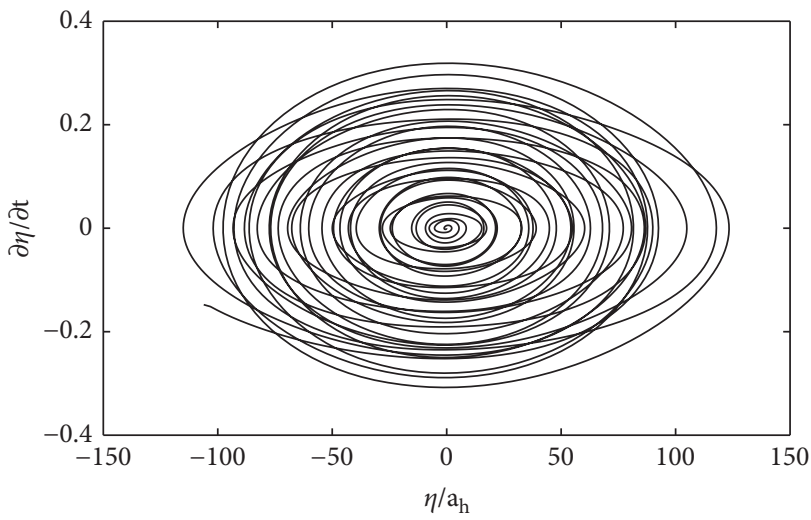

_- Analytical solution

(b)

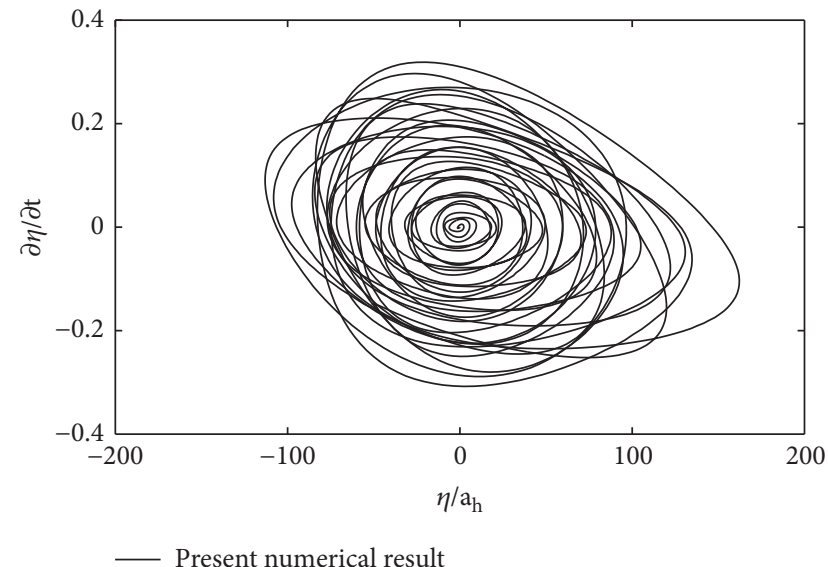

(c)

Figure 2: (a) Comparison of the nondimensional time histories of the free surface displacements at the left wall among the analytical solution (solid line), the present numerical result (dash line), and the numerical result of Frandsen [4] (circle). The phase-plane charts: (b) analytical solution and (c) present numerical result. 


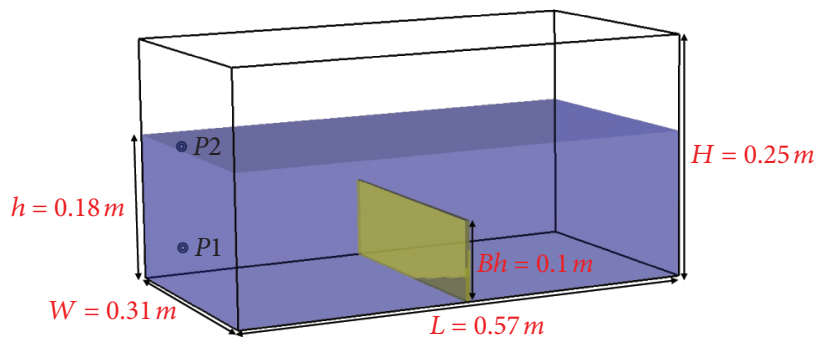

Figure 3: The sketch of the tank with a vertical baffle.

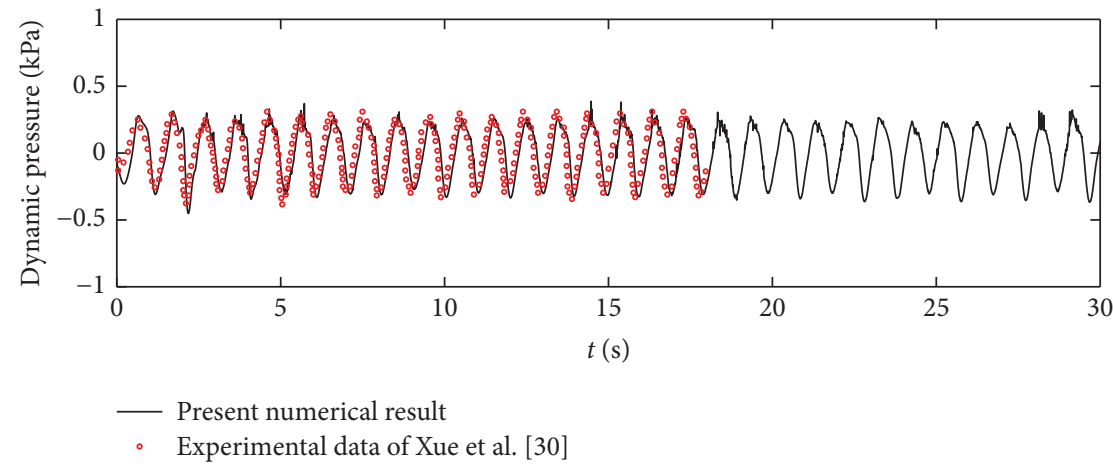

(a)

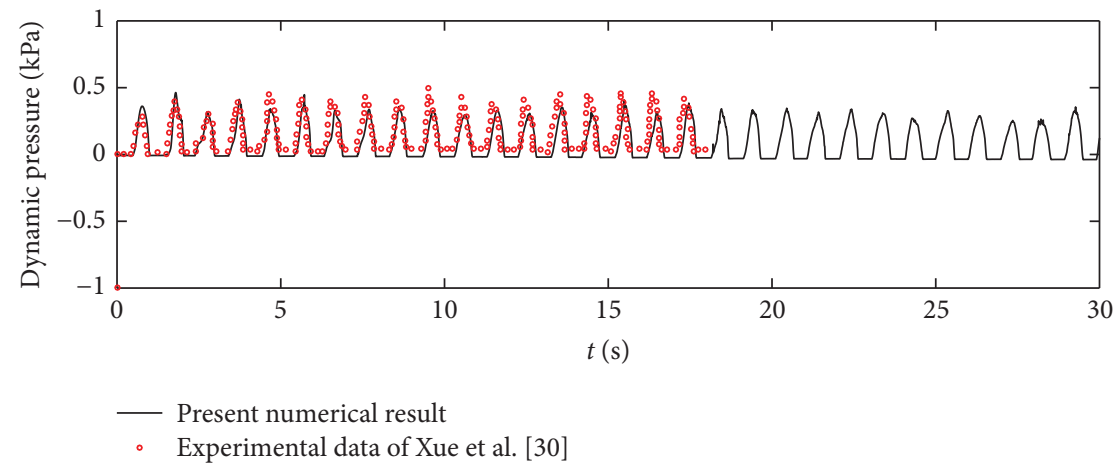

(b)

Figure 4: Comparison of the dynamic pressures at P1 (a) and P2 (b) in the tank with a vertical baffle between the present numerical result (solid line) and the experimental data of Xue et al. [30] (circle).

the upper part of the baffle to the tank bottom (we call it vertical baffle height for short), respectively. Wh denotes the length of the horizontal baffle in the tank's length direction, which is equal to $0.05 \mathrm{~m}$, and $D h$ stands for the distance of the horizontal baffle away from the tank bottom. The widths of both baffles are equal to the tank width. One wave gauge, marked by Gauge 1, is arranged at the middle of the left wall in the width direction.

Convergent tests have been conducted for different uniform grid sizes $(0.0025 \mathrm{~m}, 0.005 \mathrm{~m}$, and $0.01 \mathrm{~m})$, and the results reveal that grid size $0.005 \mathrm{~m}$ is enough to resolve the sloshing wave in baffled tanks. Then, the numerical computation domain $0.49 \mathrm{~m} \times 0.1 \mathrm{~m} \times 0.5 \mathrm{~m}$ is discretized into $98 \times 20 \times 100$ uniform grid system, with the grid sizes being $\Delta x=\Delta y=\Delta z=0.005 \mathrm{~m}$. The thickness of the baffle remains unchanged, which is equal to $0.005 \mathrm{~m}$ for all cases.
3.2. Damping Effects of Vertical Baffle. The fixed-size vertical baffle is adopted in this section, and its damping effects on coupled sloshing are discussed by changing $D v$ (Figure 5(a)). Totally, four different $D v$ are considered, $0.15 \mathrm{~m}, 0.2 \mathrm{~m}$, $0.25 \mathrm{~m}$, and $0.3 \mathrm{~m}$, resulting in the vertical baffle height $/ \mathrm{mean}$ water depth ratio $D v / h=0.5,0.67,0.83$, and 1.0 , respectively. For the case $D v / h=1.0$, it means that the baffle is arranged at the mean water depth.

The time histories of the free surface displacements and corresponding power spectral densities at Gauge 1 for different vertical baffle heights are demonstrated in the left and right columns of Figure 6, respectively. The sloshing wave in the bare tank can reach the tank roof; after the initial $12 \mathrm{~s}^{\prime}$ development, obviously steady-state envelopes appear, which is similar to heave-excited sloshing [4]. When the baffle is introduced, the wave amplitudes have a dramatic 


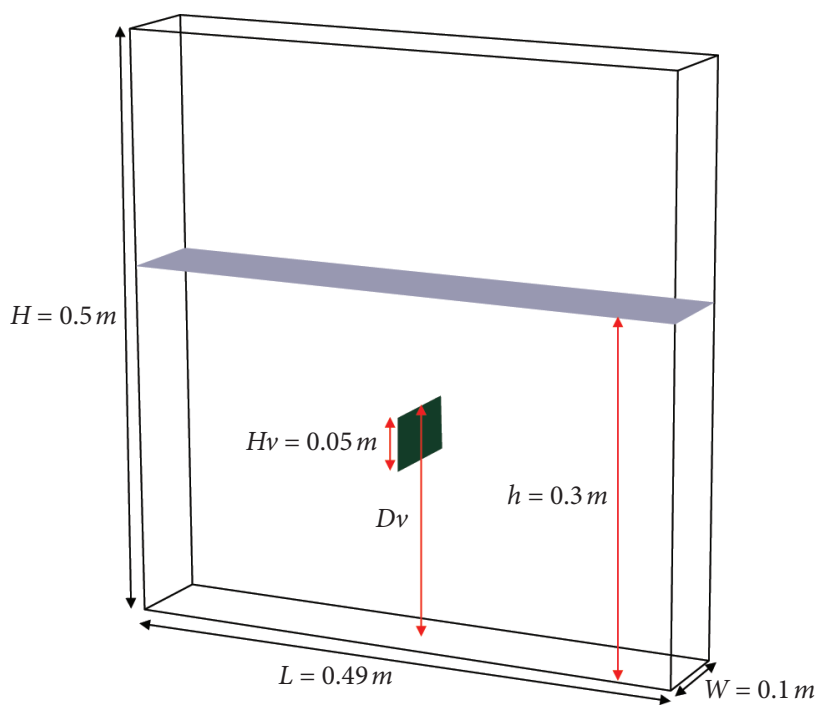

(a)

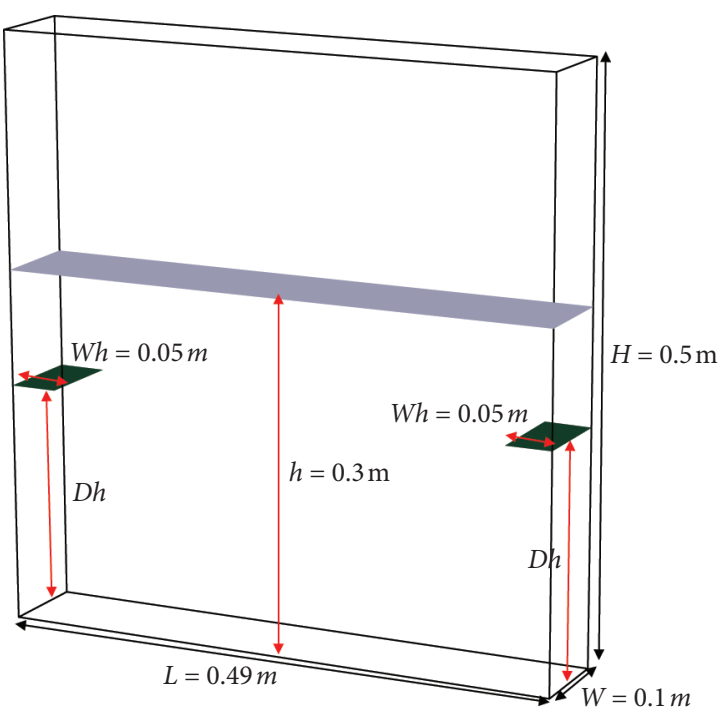

(b)

Figure 5: Baffle configurations in the present study: (a) vertical baffle and (b) horizontal baffle.
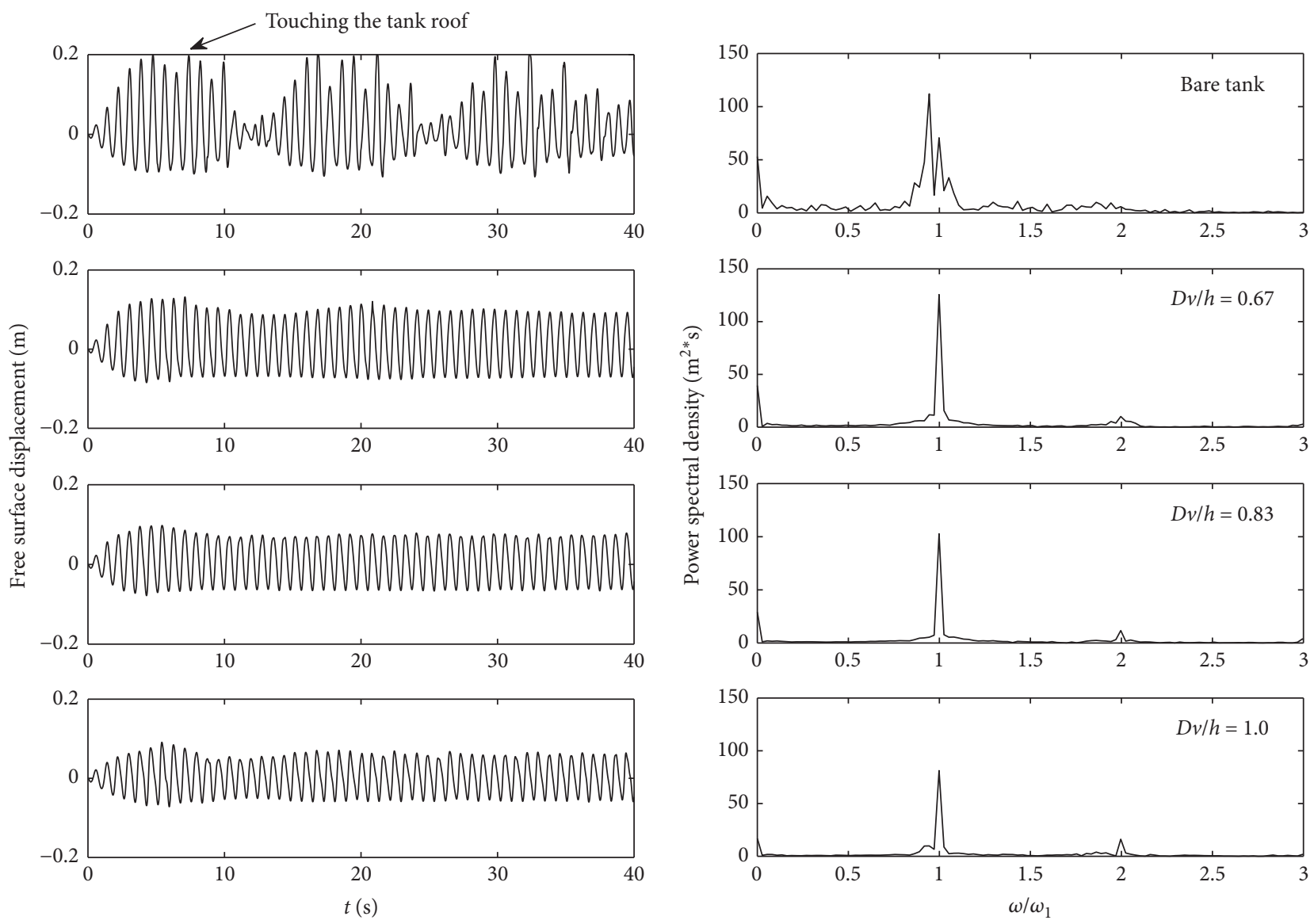

(a)

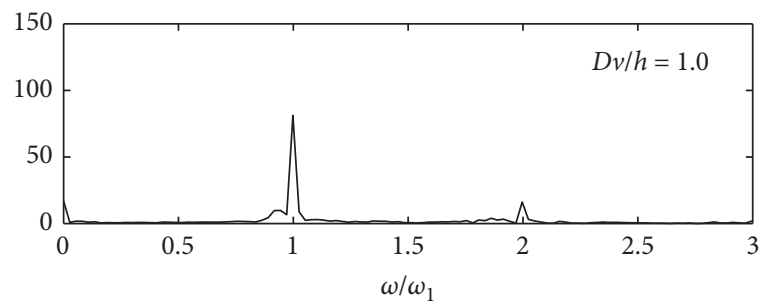

(b)

Figure 6: (a) Numerical free surface displacement and (b) the corresponding power spectra at Gauge 1 for different vertical baffle heights. 

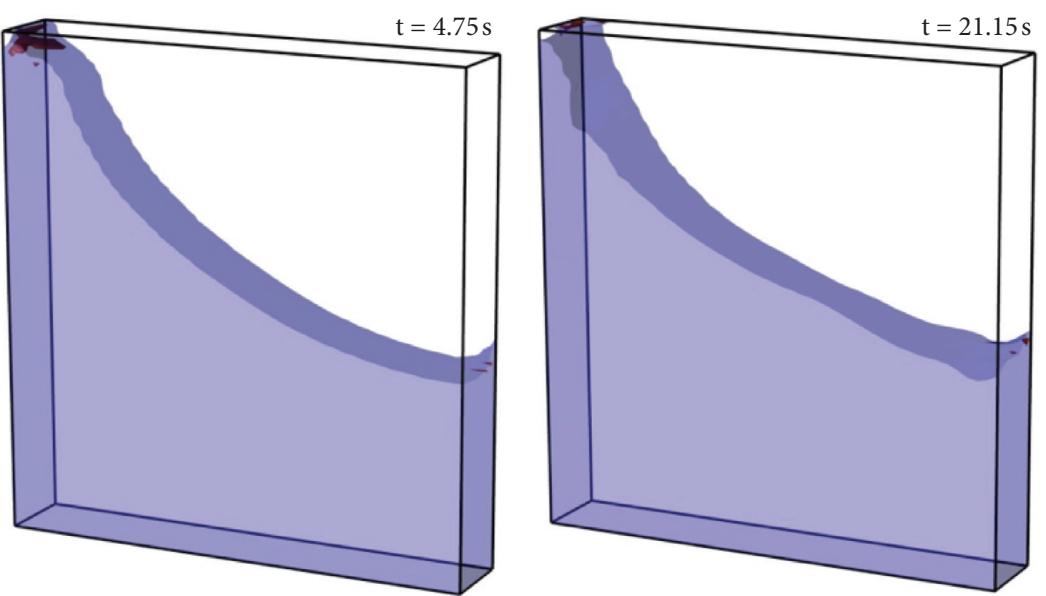

(a)

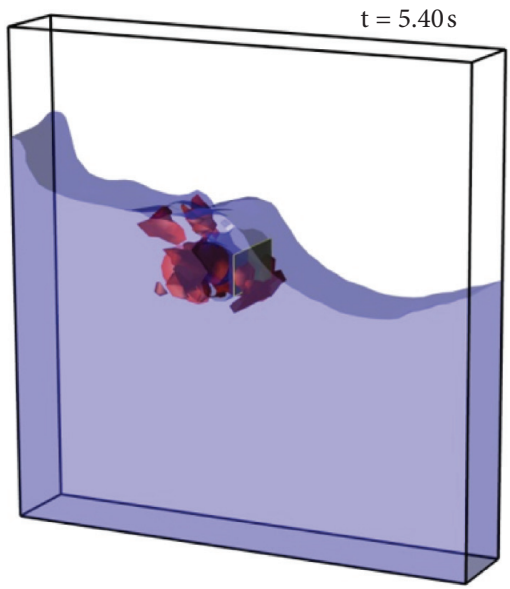

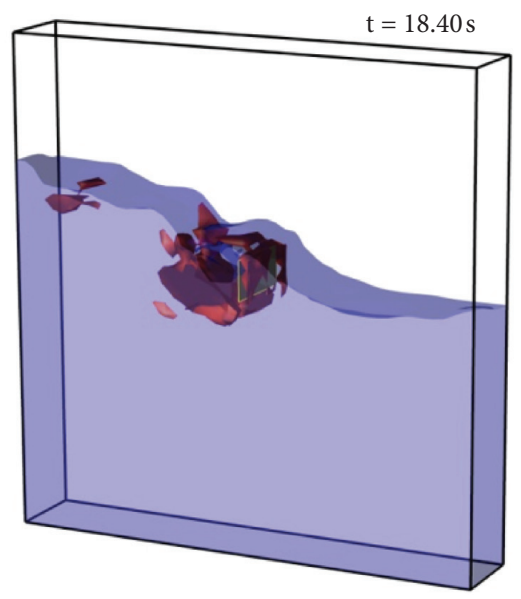

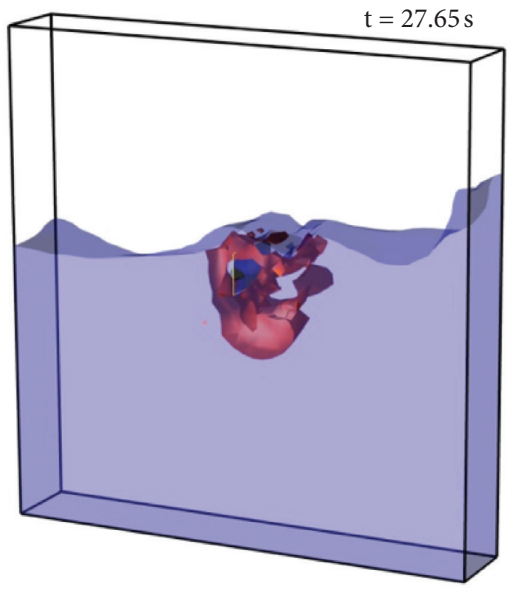

(b)

FIGURE 7: (a)Free surface snapshots and corresponding vortexes of coupled sloshing in the bare tank $(D v / h=0.0)$ and (b) tank with a vertical baffle at the mean water depth $(D v / h=1.0)$.

decrease with the increasing $D v / h$; besides, the wave gradually develops steady-state behaving as a sine function after the initial transient stage. The vertical baffle at the mean water depth is the most effective leading to maximal dissipation.

The fast Fourier transform (FFT) technique is applied to identify the frequency responses of the sloshing system, and the corresponding power spectra are illustrated in the right column of Figure 6. Despite the baffle, the dominant peak frequency of all cases is generally in accordance with $\omega_{1}$, implying the small vertical baffle has less influence on the natural frequency of the baffled system. The energy distributed at different peak frequencies in the bare tank is more scattered than other three cases that may be mainly caused by the wave breaking. The energy focused at the peak frequency decreases with the increasing $D v / h$; in other words, the vertical baffle at the free surface has maximal dissipation.

The most violent free surface snapshots of coupled sloshing in the bare tank and tank with a vertical baffle at the mean water depth and corresponding vortexes are demonstrated in Figure 7. As can be found, due to the influence of the sharper corners of the vertical baffle, strong vortexes have been created that can dissipate considerable energy. Besides, the vertical baffle can constrain the motion of the water from one side to the other, resulting in less effective water bulk climbing through the tank walls.

Figure 8 gives the relationship between $D v$ and peak free surface displacement. The vertical baffle at the mean water depth can lead to maximal dissipation compared with other cases. The dissipation of the vertical baffle for both transientstate and steady-state responses is nearly linear. When $D v$ exceeds 0.8 , the peak free surface displacement decreases inapparently, meaning the damping effect of the vertical baffle can be well when the baffle is arranged around the mean water depth.

3.3. Damping Effects of Horizontal Baffle. The damping effects of horizontal baffles are discussed in this section; the baffle dimension remains unchanged; and only $D h$ (Figure 5(b)) is systematically varied. Totally, four different $D h$ are simulated, namely, $0.15 \mathrm{~m}, 0.2 \mathrm{~m}, 0.25 \mathrm{~m}$, and $0.3 \mathrm{~m}$, with the corresponding horizontal baffle height/mean water depth ratio being $D h / h=0.5,0.67,0.83$, and 1.0 , respectively. 


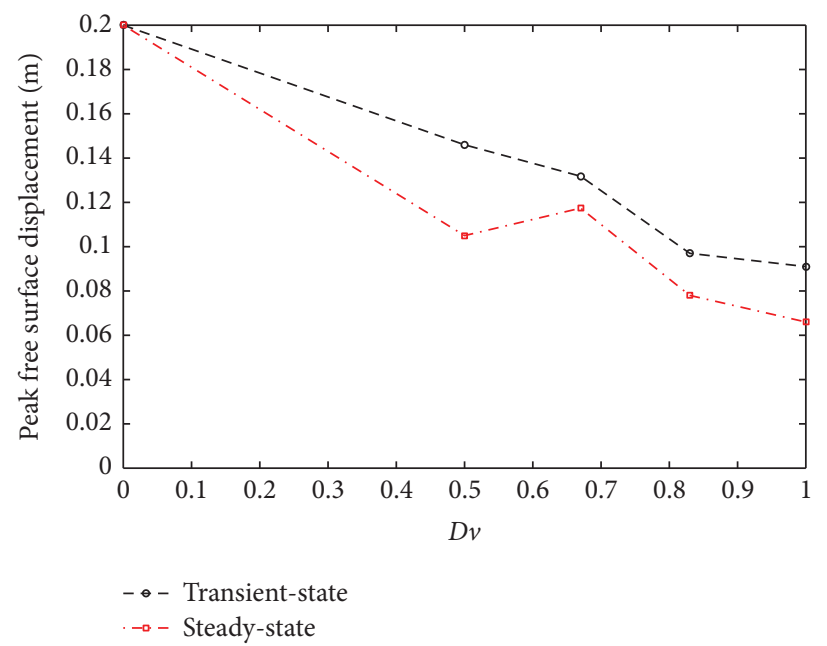

FIgURE 8: The peak free surface displacement response of coupled sloshing for different vertical baffle heights.
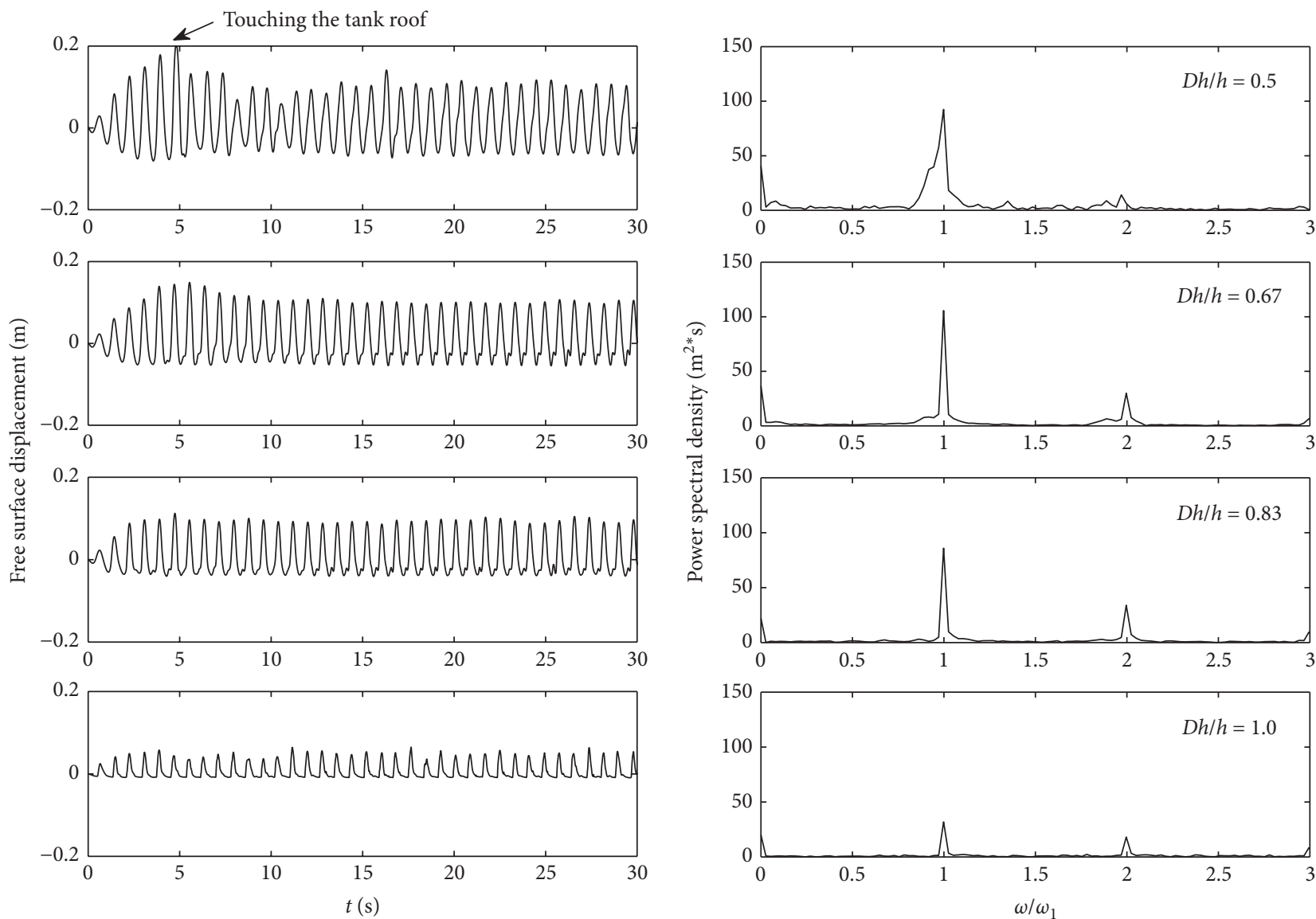

(a)

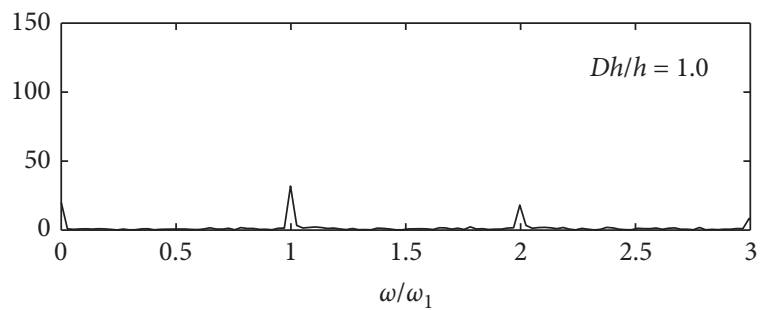

(b)

FiguRE 9: (a) Numerical free surface displacement and (b) the corresponding power spectra at Gauge 1 for different horizontal baffle heights.

Similar to the former vertical case $D v / h=1.0$, the present case with $D h / h=1.0$ means that the horizontal baffle is installed at the mean water depth.

Figure 9 gives the time histories of the free surface displacements $(9(\mathrm{a}))$ and corresponding power spectral densities (9(b)) at Gauge 1 for different horizontal baffle heights. For the case that the horizontal baffles are arranged at a lower level $(D h / h=0.5)$, the water can hit the ceiling and then begin to break. As the baffle height reaches $0.2 \mathrm{~m}$, correlated to $D h / h=0.67$, the wave decreases obviously 

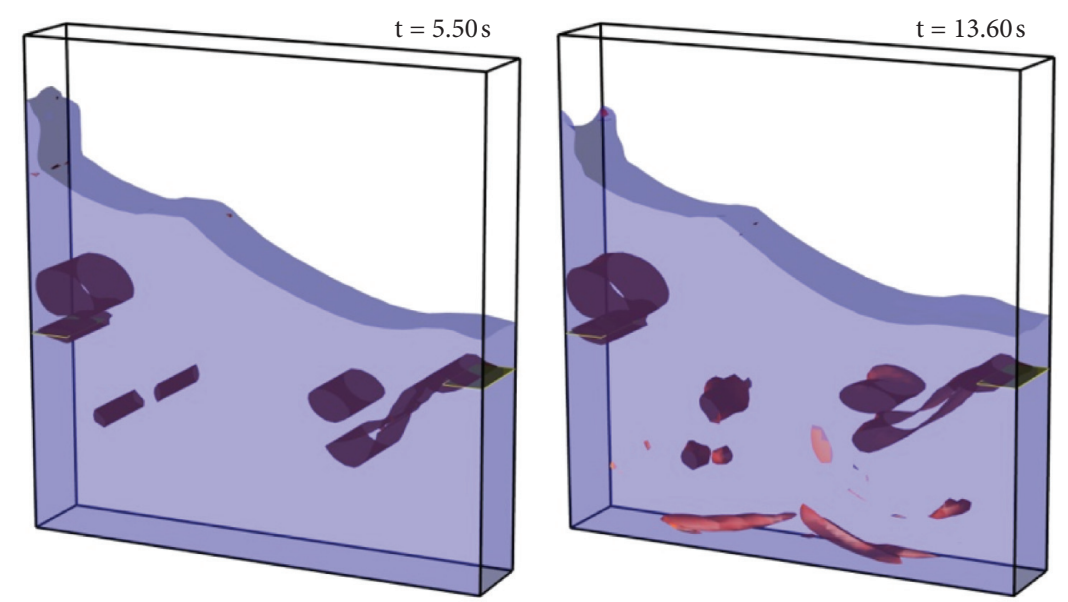

(a)
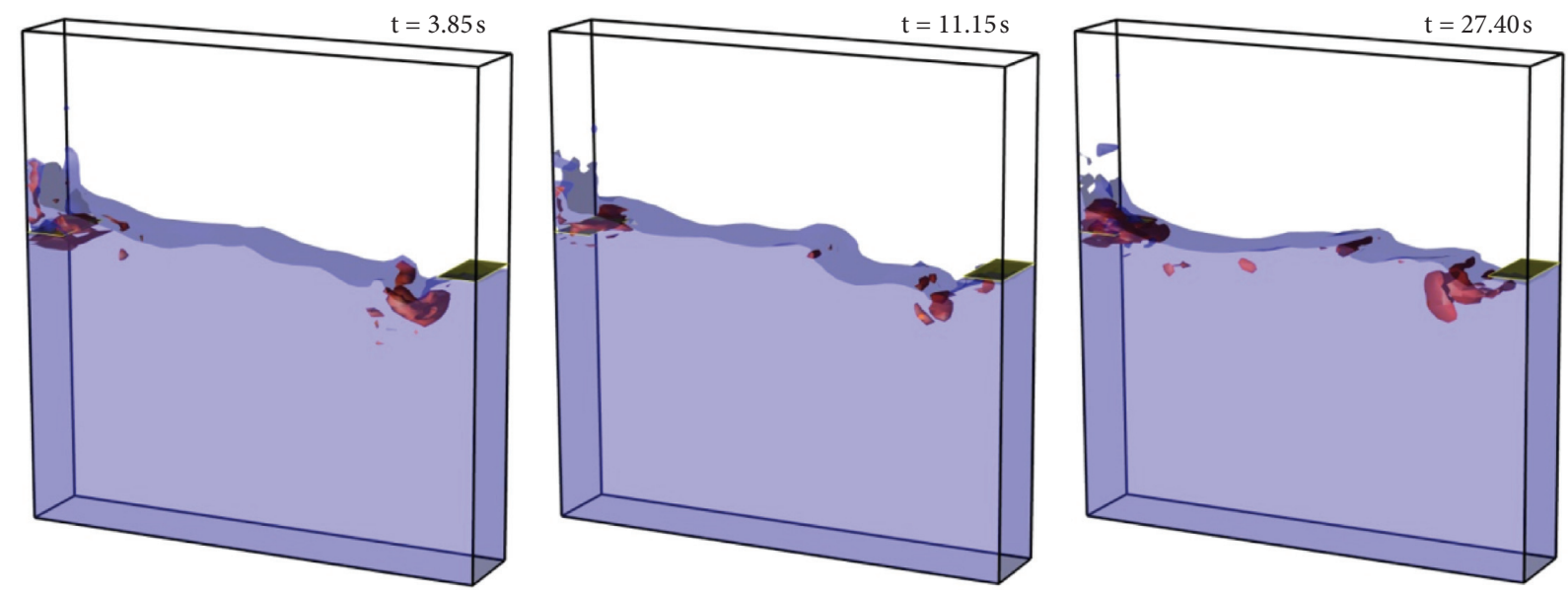

(b)

FIGURE 10: Free surface snapshots and the vortexes of coupled sloshing for various horizontal baffle heights: (a) $D h / h=0.67$ and (b) $D h / h=1.0$.

compared with the case of $D h / h=0.5$; besides, after the first $5.5 \mathrm{~s}$ transient development, the wave comes to be steadystate. If the baffle height is extensively increased, the response wave can develop into a steady state rapidly. Similar to that of the vertically baffled tank, the horizontal baffle arranged at the mean water depth $(D h / h=1.0)$ is also the most effective.

The corresponding power spectra results reveal the dominant peak frequencies of all cases are still $\omega_{1}$, and the secondary peak frequencies are $2 \omega_{1}$, meaning the dominant wave is the first mode and the small structure has limited influences on the natural frequency of the system. Obviously, the energy focused at the dominant peak frequency decreases with the increasing $D h / h$, among which the horizontal baffles at the free surface can lead to maximal dissipation, and mild wave motion can be aroused. The most violent free surface snapshots and corresponding vortexes for various horizontal baffle heights $(D h / h=0.67$ and 1.0$)$ are

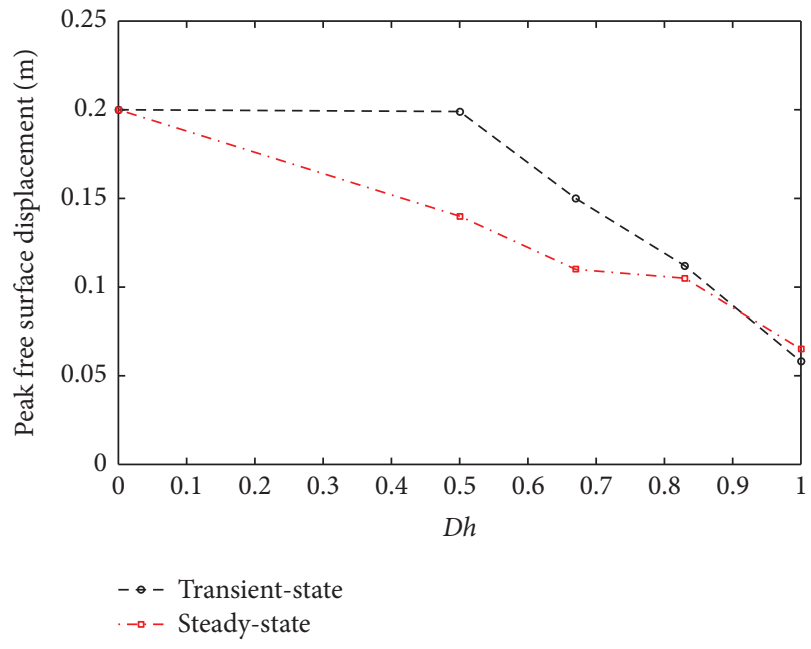

FIGURE 11: The peak free surface displacement response of coupled sloshing for different horizontal baffle heights. 
demonstrated in Figure 10. Similar to the vertical baffle cases in Figure 7, strong vortexes have been generated by the sharper corners of the baffles that can result in considerable energy dissipation. Moreover, the vertical motion can be constrained by the horizontal baffle at both sides leading to less water can move freely through the tank walls.

Figure 11 gives the relationship between $D h$ and peak free surface displacement. Similar to that in the tank with a vertical baffle, the horizontal baffles at the mean water depth have maximal dissipation, leading to a peak value being about $0.06 \mathrm{~m}$, which is equal to $0.2 \mathrm{~h}$. The dissipation of the horizontal baffles for both transient-state and steady-state responses is generally linear when $D v$ exceeds 0.5. Overall, from the above two categories of baffles, we can conclude that the baffle around the mean water depth works better than other cases.

\section{Conclusions}

A Navier-Stokes model was adopted to study liquid sloshing in a rectangular tank under coupled surge and heave excitations. After full validations against the available numerical, theoretical, and experimental results, the numerical model was used to research the fieriest coupled sloshing where the surge and heave excitation frequencies were the first-order natural frequency and twice of the firstorder natural frequency, respectively. In order to reduce the fieriest sloshing wave that was strongly broken, two kinds of baffles, namely, vertical and horizontal baffles, were used to expect to suppress the insecure wave. Four different baffle positions were considered, and the results revealed that the baffle at the mean water depth was the most effective in suppressing the violent sloshing wave. Besides, the horizontal baffles at the mean water depth worked better than the vertical baffle. The damping mechanism was divided into two parts: one was vortex dissipation caused by the sharper corners of the baffles, and the other was the reduction of the effective water bulk climbing through the tank walls.

\section{Data Availability}

No data are available on the account of the in-house numerical model.

\section{Conflicts of Interest}

The authors declare that they have no conflicts of interest.

\section{Authors' Contributions}

Ren Lv and Xin Jin conceptualized the study. Ren Lv developed the methodology. Ren Lv, Yinjie Zou, Jinbo Tang, Dengsong Li, and Mingming Liu performed validation. Ren Lv, Yinjie Zou, and Jinbo Tang performed visualization. Ren Lv and Jinbo Tang wrote the original draft. Xin Jin Mingming Liu reviewed and edited the article. Xin Jin supervised the study.

\section{Acknowledgments}

The work was supported by the National Natural Science Foundation of China (Grant no. U20A20112), Open Fund Projects of State Key Laboratory of Oil and Gas Reservoir Geology and Exploitation (Grant no. PCL2020011), State Key Laboratory of Hydraulic Engineering Simulation and Safety (Grant no. HESS-2009), and Key Laboratory of Ministry of Education for Coastal Disaster and Protection (Grant no. 202005).

\section{References}

[1] W. Zhao, J. Yang, Z. Hu, and L. Tao, "Coupled analysis of nonlinear sloshing and ship motions," Applied Ocean Research, vol. 47, pp. 85-97, 2014.

[2] O. M. Faltinsen, "A numerical nonlinear method of sloshing in tanks with two-dimensional flow," Journal of Ship Research, vol. 22, no. 3, pp. 193-202, 1978.

[3] O. M. Faltinsen and A. N. Timokha, "An adaptive multimodal approach to nonlinear sloshing in a rectangular tank," Journal of Fluid Mechanics, vol. 432, pp. 167-200, 2001.

[4] J. B. Frandsen, "Sloshing motions in excited tanks," Journal of Computational Physics, vol. 196, no. 1, pp. 53-87, 2004.

[5] O. M. Faltinsen and A. N. Timokha, Sloshingpp. 1-606, Cambridge University Press, Cambridge, UK, first edition, 2009.

[6] R. A. Ibrahim, "Recent advances of structural life assessment and related problems," Springer Proceedings in Physics, vol. 137, pp. 1-27, 2015.

[7] O. M. Faltinsen, "A nonlinear theory of sloshing in rectangular tanks," Journal of Ship Research, vol. 18, no. 4, pp. 224-241, 1974.

[8] T. B. Benjamin and F. Ursell, "The stability of the plane free surface of a liquid in vertical periodic motion," Proceedings of the Royal Society A: Mathematical, Physical and Engineering Sciences, vol. 225, no. 1163, pp. 505-515, 1954.

[9] O. M. Faltinsen, O. F. Rognebakke, I. A. Lukovsky, and A. N. Timokha, "Multidimensional modal analysis of nonlinear sloshing in a rectangular tank with finite water depth," Journal of Fluid Mechanics, vol. 407, pp. 201-234, 2000.

[10] O. M. Faltinsen, O. F. Rognebakke, A. N. Timokha, and A. N. Timokha, "Resonant three-dimensional nonlinear sloshing in a square-base basin," Journal of Fluid Mechanics, vol. 487, pp. 1-42, 2003.

[11] O. M. Faltinsen, O. E. Lagodzinskyi, and A. N. Timokha, "Resonant three-dimensional nonlinear sloshing in a square base basin. Part 5. Three-dimensional non-parametric tank forcing," Journal of Fluid Mechanics, vol. 894, p. A10, 2020.

[12] M.-A. Xue and P. Lin, "Numerical study of ring baffle effects on reducing violent liquid sloshing," Computers and Fluids, vol. 52, pp. 116-129, 2011.

[13] M. Luo, C. G. Koh, and W. Bai, "A three-dimensional particle method for violent sloshing under regular and irregular excitations," Ocean Engineering, vol. 120, pp. 52-63, 2016.

[14] Z. Ozdemir, Y. M. Fahjan, and M. Souli, "Numerical simulation of liquid sloshing," in Computational Methods in Earthquake Engineering, pp. 49-79, Springer, Cham, Switzerland, 2017.

[15] H. Zhu, Y. Gao, N. Srinil, and Y. Bao, "Mode switching and standing-travelling waves in slug flow-induced vibration of catenary riser," Journal of Petroleum Science and Engineering, vol. 203, Article ID 108310, 2021. 
[16] T. Nakayama, "Boundary element analysis of nonlinear water wave problems," International Journal for Numerical Methods in Engineering, vol. 19, no. 7, pp. 953-970, 1983.

[17] H. M. Koh, J. K. Kim, and J.-H. Park, "Fluid-structure interaction analysis of 3-D rectangular tanks by a variationally coupled BEM-FEM and comparison with test results," Earthquake Engineering and Structural Dynamics, vol. 27, no. 2, pp. 109-124, 1998.

[18] B. Godderidge, S. Turnock, M. Tan, and C. Earl, "An investigation of multiphase CFD modelling of a lateral sloshing tank," Computers and Fluids, vol. 38, no. 2, pp. 183-193, 2009.

[19] M.-A. Xue, Y. Chen, J. Zheng, L. Qian, and X. Yuan, "Fluid dynamics analysis of sloshing pressure distribution in storage vessels of different shapes," Ocean Engineering, vol. 192, Article ID 106582, 2019.

[20] D. Ning, W.-H. Song, Y.-L. Liu, and B. Teng, “A boundary element investigation of liquid sloshing in coupled horizontal and vertical excitation," Journal of Applied Mathematics, vol. 201220 pages, 2012, Special Issue, Article ID 340640.

[21] B. Chen and R. Nokes, "Time-independent finite difference analysis of fully non-linear and viscous fluid sloshing in a rectangular tank," Journal of Computational Physics, vol. 209, no. 1, pp. 47-81, 2005.

[22] W. Bai, X. Liu, and C. G. Koh, "Numerical study of violent LNG sloshing induced by realistic ship motions using level set method," Ocean Engineering, vol. 97, pp. 100-113, 2015.

[23] M. Luo, X. Wang, X. Jin, and B. Yan, "Three-dimensional sloshing in a scaled membrane LNG tank under combined roll and pitch excitations," Ocean Engineering, vol. 211, Article ID 107578, 2020.

[24] S. M. Hasheminejad and M. Aghabeigi, "Sloshing characteristics in half-full horizontal elliptical tanks with vertical baffles," Applied Mathematical Modelling, vol. 36, no. 1, pp. $57-71,2012$.

[25] S. K. Nayak and K. C. Biswal, "Fluid damping in rectangular tank fitted with various internal objects - an experimental investigation,” Ocean Engineering, vol. 108, pp. 552-562, 2015.

[26] R. Yuan, D. B. Meng, and H. Q. Li, "Multidisciplinary reliability design optimization using an enhanced saddlepoint approximation in the framework of sequential optimization and reliability analysis," Proceedings of the Institution of Mechanical Engineers, Part O: Journal of Risk and Reliability, vol. 230, no. 6, pp. 570-578, 2017.

[27] I. H. Cho, J.-S. Choi, and M. H. Kim, "Sloshing reduction in a swaying rectangular tank by an horizontal porous baffle," Ocean Engineering, vol. 138, pp. 23-34, 2017.

[28] M.-A. Xue and O. Kargbo, "Seiche oscillations of layered fluids in a closed rectangular tank with wave damping mechanism," Ocean Engineering, vol. 196, p. 106842, 2020.

[29] H. Akyildiz and E. Ünal, "Experimental investigation of pressure distribution on a rectangular tank due to the liquid sloshing," Ocean Engineering, vol. 32, pp. 1503-1516, 2005.

[30] M. A. Xue, J. H. Zheng, P. Lin, and X. L. Yuan, "Experimental study on vertical baffles of different configurations in suppressing sloshing pressure," Ocean Engineering, vol. 136, pp. 178-189, 2017.

[31] Y. Chen and M. A. Xue, "Numerical simulation of liquid sloshing with different filling levels using OpenFOAM and experimental validation," Water (Switzerland), vol. 10, p. 1752, 2018.

[32] H. Zhu, W. Liu, and T. Zhou, "Direct numerical simulation of the wake adjustment and hydrodynamic characteristics of a circular cylinder symmetrically attached with fin-shaped strips," Ocean Engineering, vol. 195, Article ID 106756, 2020.
[33] M.-A. Xue, Z. Jiang, Y.-A. Hu, and X. Yuan, "Numerical study of porous material layer effects on mitigating sloshing in a membrane LNG tank," Ocean Engineering, vol. 218, Article ID 108240, 2020.

[34] V. S. Sanapala, K. Velusamy, and B. S. V. Patnaik, "Numerical simulation of parametric liquid sloshing in a horizontally baffled rectangular container," Journal of Fluids and Structures, vol. 76, pp. 229-250, 2018.

[35] H. Saghi, D. Ning, P. Cong, and M. Zhao, "Optimization of baffled rectangular and prismatic storage tank against the sloshing phenomenon," China Ocean Engineering, vol. 34, pp. 1-13, 2020.

[36] H. Jin, A. Calabrese, and Y. Liu, "Effects of different damping baffle configurations on the dynamic response of a liquid tank under seismic excitation," Engineering Structures, vol. 229, p. 111652, 2021.

[37] D. Liu and P. Lin, "A numerical study of three-dimensional liquid sloshing in tanks," Journal of Computational Physics, vol. 227, pp. 3921-3939, 2008.

[38] J. B. Frandsen and W. Peng, "Experimental sloshing studies in sway and heave base excited square tanks," in ASCE Proceedings of the Civil Engineering in the Oceans VI, Baltimore, MA, USA, October 2005.

[39] X. Jin, "Experimental and numerical investigation of the effect of liquid viscosity on sloshing characteristics under horizontal and vertical excitations," Ph.D. thesis, Sichuan University, Chengdu, China, 2018. 